\title{
OPERATION OF THE LOW-ENERGY DEMONSTRATION ACCELERATOR: THE PROTON INJECTOR FOR APT*
}

\author{
J. David Schneider $\approx$, Los Alamos National Laboratory
}

\section{Abstract}

We report preliminary test results for a $6.7-\mathrm{MeV}, 100-\mathrm{mA}$ cw radio-frequency quadrupole. During the time this structure was in design, fabrication, assembly, tuning, and installation, we tested a $75-\mathrm{keV}$ proton injector, $350-\mathrm{MHz}$ high-power RF systems, EPICS controls, diagnostics, beam stop, and other hardware in preparation for the 670$\mathrm{kW}$ beam test. Initial LEDA RFQ beam operation has commenced. This paper summarizes work in the past year, concentrating on RFQ assembly, tuning, installation, high-power conditioning and initial beam operation. We will include summaries of injector tests, 1.25-MeV RFQ-beam tests, beam-transport and beam-stop performance, and cavity resonance control. The technology being developed in this first section of the APT (accelerator production of tritium) linac is also appropriate for several other applications, including the transmutation of waste and medical isotope production.

\section{LEDA DESCRIPTION}

\subsection{Purpose and Background}

LEDA is designed to be a complete prototype[1] of the front end of the APT accelerator. Within LEDA, we plan to build, test, and characterize the injector, RFQ, first section of CCDTL, and all necessary interface components to help prove the feasibility of the APT 100-mA, cw linac. At this stage, the injector and RFQ (Fig 1) are functional and undergoing initial integrated beam testing.

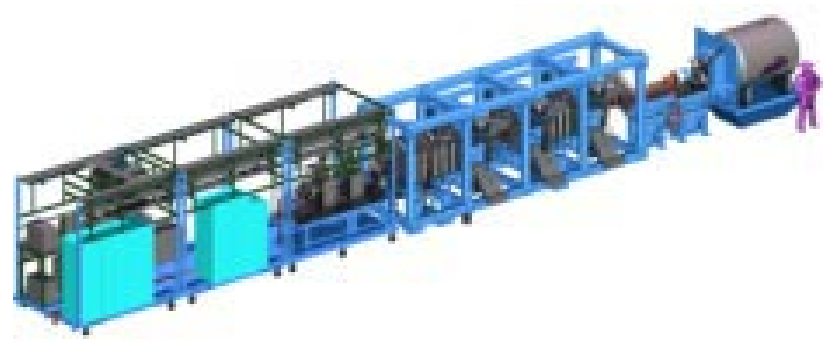

Figure 1: Computer rendering of LEDA, showing injector and its electronics on left, RFQ inside frame, and short transport line and beam stop.

\subsection{Injector}

The unique LEDA injector[2] uses an ion source that is a slightly modified version of a simple and effective microwave-driven proton source developed at Chalk River
Laboratory in Ontario. One unique feature of this source is that no electronics reside at high potential. Even the electromagnetic solenoids are at ground potential, separated from the source chamber by a specially designed polypropylene insulator. Microwave power is fed through an insulated waveguide break, allowing the $2-\mathrm{kW}, 2.45-$ $\mathrm{GHz}$ power source to be at ground potential. Similarly, the hydrogen gas feed is through a small-diameter insulated tube kept at near atmospheric pressure to suppress breakdown.

The extractor is a $75-\mathrm{kV}$ single-gap, shaped-surface configuration, with integral electron suppression. Both triode and tetrode versions have been tested[3]. The transport line[4] is about $2.7 \mathrm{~m}$ long, and includes two electromagnetic solenoids for focusing, and two pairs of dual-axis steerers. Variable and insertable ring collimators are used for additional beam-current control. Vacuum pumping and several diagnostic devices (mostly noninterceptive) are included in this transport line.

This injector configuration (Fig.2) has been tested[5] since 1992, demonstrating consistently excellent performance, including injection into a cw RFQ[6]. For example, this injector can provide more than $130 \mathrm{~mA}$ of ion current, with $90 \%$ proton fraction, at a rms normalized emittance of $0.19 \pi \mathrm{mm}$ mrad. The LEDA injector sits on rail-mounted platforms to facilitate easy movement to the RFQ. Strict attention to layout and grounding guarantees that neither equipment damage nor computer upsets result from infrequent high-voltage sparkdowns[7].

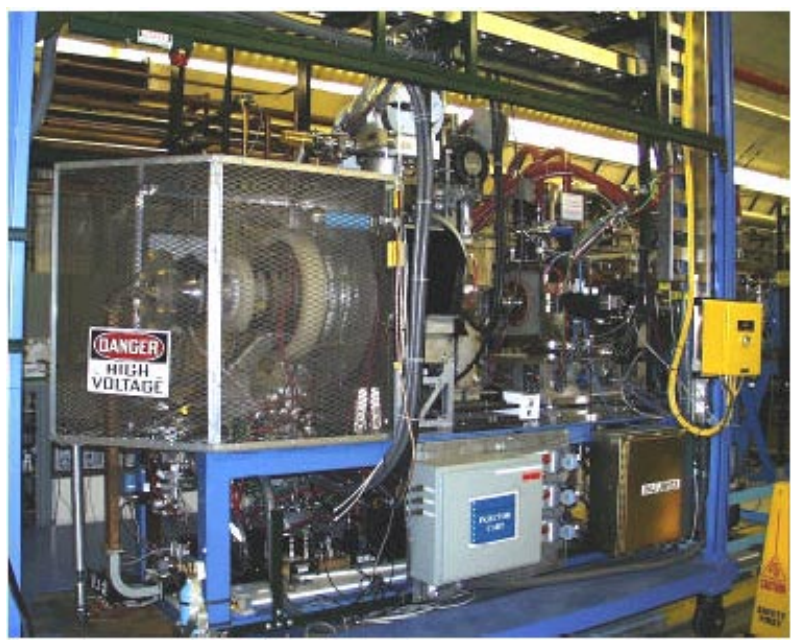

Figure 2. Beamline hardware of the LEDA Injector.

* Work supported by the US DOE, Defense Programs

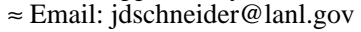




\subsection{Radio-Frequency Quadrupole}

The LEDA RFQ must provide excellent performance under very demanding $\mathrm{cw}$ operation[8]. Output energy is $6.7 \mathrm{MeV}$, with proton injection at $75 \mathrm{keV}$. Total length is approximately 8 meters, comprised of eight sections to facilitate brazing. There are four 2-m-long resonant segments to facilitate RF tuning of this structure. After precise alignment during brazing and assembly, 128 static slug tuners are used to ensure proper field distribution and to reduce non-quadrupole fields to less than $2 \%$ of the primary field. During operation, structure resonance is maintained by precise automatic adjustment of the cooling water temperature[9]. Temperature control of the RFQ copper structure controls cavity dimensions and resonant frequency. Design peak fields for this cw RFQ are 1.8 Kilpatrick or $33 \mathrm{MV} / \mathrm{m}$ at $350 \mathrm{MHz}$.

All major pieces of the RFQ resonant structure are made of OFE copper. Some flanges are made of GlidCop $^{\mathrm{TM}}$, a strengthened version of OFE, fortified with a suspension of aluminum oxide powder to increase hardness.

A major engineering challenge on this RFQ was to properly support this 8-m long, cylinder of annealed OFE copper. The weight $(2360 \mathrm{~kg}$ ) of the RFQ is supported by five struts, attached to the flanges separating the twometer-long segments. The outer ends of these struts attach to a very study steel frame, that in turn is held above the floor by three kinematic mounts. A similar set of seven horizontal struts constrain the RFQ in transverse and longitudinal motion.

All other RFQ-related hardware, including windows, vacuum and water manifolds, and vacuum waveguide sections, are separately suspended from the outer steel frame, to avoid putting undue stress onto the RFQ copper.

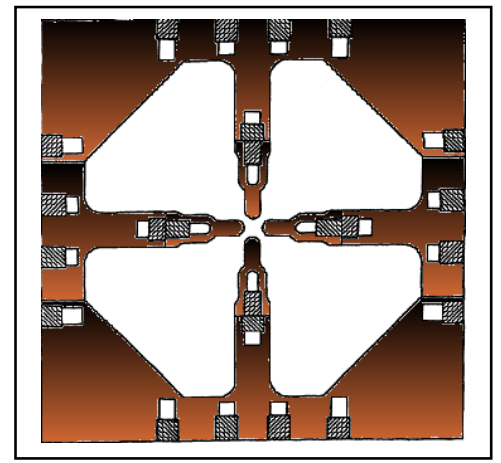

Figure 3. Cross section of the LEDA RFQ, showing locations of the 24 water cooling channels in each of the eight sections.

Three of the eight RFQ sections have provisions for balanced RF power feeds, one into each quadrant of that section. Well-cooled, tapered, ridged vacuum waveguides carry this power to a coupling slot in the outer wall of the RFQ. Each coupling slot (Fig.4) is approximately 1.7 $\mathrm{mm}$ wide, by $8.9 \mathrm{~cm}$ long. A coaxial RF window is used to separate atmospheric WR2300 waveguide from a halfheight vacuum waveguide (Fig. 5).

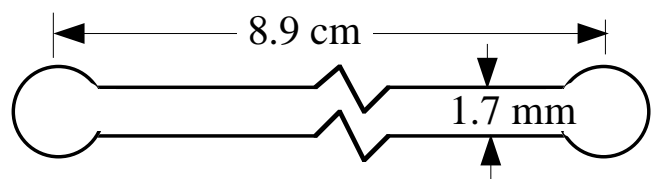

Figure 4. Geometry of RF coupling iris, through which RF power enters the LEDA RFQ.

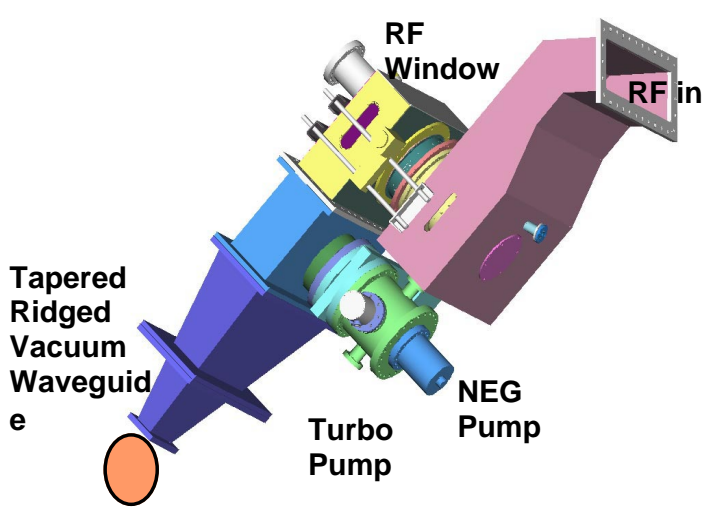

Figure 5. Depiction of the RF window assembly for feeding power into the RFQ.

All vacuum systems[10] used on LEDA are oil-free to minimize the chance of internal oil contamination on accelerating structures. Cryogenic pumps are used for the RFQ cavity, and non-evaporative getter (NEG) pumps maintain an a high-vacuum environment for the RF windows. Turbo-molecular pumps provide additional pumping for all structures.

\subsection{RF Power}

LEDA's RF power system[11] includes a number of both $350-\mathrm{MHz}$ and $700-\mathrm{MHz} \mathrm{cw}$ transmitters. Three of the 350$\mathrm{MHz}$ systems are dedicated for use on the RFQ. Although each $350-\mathrm{MHz}$ RF klystron is rated for, and has demonstrated, 1.3 MW of supplied power, losses, mismatches, and control margin reduce the effective perklystron RFQ-delivered power to just under 1.0 MW. Ohmic losses in the walls of the RFQ are approximately $1.2 \mathrm{MW}$, and absorbed beam power is about $0.67 \mathrm{MW}$. Two klystrons should thus provide all power needed for full-current operation, but this entails running the klystrons very near maximum levels. The third klystron was included to ensure that we have adequate operational power margin. We are now running $2 \mathrm{RF}$ systems into the RFQ using the accelerating cavity as a power combiner. The third 350-MHz RF system is ready for use, but is not presently attached to the RFQ.

A nominal $1 \mathrm{MW}$ of $350-\mathrm{MHz}$ RF power from each klystron is carried through WR1500 waveguide about 40 meters from the klystron, to a magic-tee power splitter, and then split again. Power from each klystron[12] is split 
equally into four feeds, each of which enters a quadrant of the RFQ. Commercial coaxial RF windows are used to separate the atmospheric waveguide from the stepped, ridged vacuum waveguide transitions that feed this power to the coupling irises on the RFQ outer wall. These commercial RF windows were tested[13] at sustained $\mathrm{cw}$ power levels of $1 \mathrm{MW}$, in preparation for use on the RFQ at less than 0.34 MW.

LEDA's specialized low-level RF (LLRF) system has a number of functions[14], including maintaining correct power and phase in the cavity fields. Controller boards for these functions use I \& Q control[15] rather than direct amplitude and phase. A combination of digital and analog control loops is used to provide both long-term precision and fast response.

The LLRF system must maintain the RFQ cavity on resonance. This is done by sending a signal to the resonance-control cooling system (RCCS) that in turn makes adjustments to the cooling water temperature at the RFQ vane bases. Cooling water temperature is the only dynamically adjustable parameter for the RFQ. Protection against internal arcs and faults both in the RFQ cavity and in the several RF windows near the RFQ are additional functions provided by the LLRF system.

\subsection{Beam Stop and Transport Line}

The innovative beam stop is described previously[16] Key features of this design are its compactness, light-weight, integral neutron shielding, low radiation source term, simplicity, and ease of replacement or removal. Audio sensors on the cartridge should sense any unexpected water boiling well before thermal failure of the structure.

A smaller prototype was demonstrated with a $125-\mathrm{kW}$ beam from the CRITS RFQ. A LEDA beam-stop replacement cartridge is under development now at General Atomics that includes an internal carbon liner to further reduce the prompt and residual radiation source term.

The high-energy beam transport (HEBT) line[17] is as simple and short $(2.5 \mathrm{~m})$ as feasible, includes five quadrupoles, two dual-axis beam steerers, vacuum pumping, and a number of beam diagnostic devices[18].

\subsection{Facilities Upgrades}

When LEDA assumed occupancy of an existing building at Los Alamos, that facility had only about $3 \mathrm{MW}$ of ac power and cooling capacity. In order to operate up to six RF transmitters and other equipment, LEDA needs nearly $15 \mathrm{MW}$ of power. We upgraded the raw ac power and distribution system and installed ten specialized cooling systems needed for all accelerating structures and related equipment.

This upgrade activity required as many as 85 crafts people working adjacent to a number of experimentalists. In this process, the LEDA project accumulated more than 220,000 construction man-hours with only a single minor lost-time accident.

\subsection{Controls}

The EPICS[19] system is used for all integrated controls on LEDA, although LabView is often used for off-line development of individual subsystems. In several instances, e.g. high-power RF and vacuum systems, a dedicated PLC (programmable logic controller) is used to facilitate local operation and to handle most interlocks. But, during integrated system operation from the control room, EPICS provides operator tools and links to nearly all read-back and control channels.

\subsection{Safety and Protection Systems}

The primary personnel safety system on LEDA is the personnel access control system (PACS), whose function is to ensure that personnel are excluded from the beam tunnel's potentially high radiation areas during beam operation. This LEDA safety system represents the best version of the PACS units used on LANSCE. All PACS wiring is in protected conduits, all switches are redundant and tamper-proof, all logic (in redundant, locked boxes) is via a dedicated PLC, and strict administrative controls are used for all installation, checkout, and routine use.

A backbone beam enable (BBE) hard-wired circuit is implemented in a fashion very similar to the PACS. This system ensures that beam operation is inhibited whenever hardware configurations are outside the safe operating boundary. One input example is the water level in a neutron shield tank surrounding the beam stop.

A hard-wired fast-protect system is used to interrupt beam current within a few micro-seconds in the event of a of a malfunction that indicate possible beam misbehavior.

A large fraction of the instrumented channels on LEDA are monitored by the beam run-permit system. This equipment-protection function is done in EPICS software, and may have response times approaching one second. However, its purpose is to ensure that beam delivery is permitted only when all critical systems are in proper operation and alignment.

Of course, many hard-wired interlocks are used extensively throughout LEDA. Adequate water flow in various electromagnets and valve positions are typical examples.

The LEDA Accelerator Readiness Assessment (ARA) process included a simultaneous review by an independent contractor team and a DOE team. This process, although demanding for a one-week period, worked extremely well. This readiness review was done the first week in December, 1998; only three working days after we were able to initiate RFQ conditioning with a single 1-MW klystron. Closeout of the ARA process was completed on March 12, two working days before our first RFQaccelerated beam.

All our documentation and operational approval processes have been expedited by early and fruitful collaboration with all parties, especially with DOE, our sponsor for this work. 


\section{BEAM TESTING}

\subsection{RFQ Conditioning Notes}

Initial conditioning[20] was done with a single klystron feeding into the four ports of segment $\mathrm{B}$, with segment $\mathrm{C}$ ports blanked off, and waveguide terminations properly positioned outside segment D windows. All subsequent conditioning has been done with two phase-locked 1-MW klystrons, feeding into segments B and D.

At very low power levels (a few $\mathrm{kW}$ ) we saw the expected initial multipacting in all window assemblies. However, some amount of multipacting persisted to higher-than-expected power levels. With four windows installed, we saw multipacting up to powers of about 400 $\mathrm{kW}$. With eight windows, multipacting continued to about $800 \mathrm{~kW}$. Thus we conclude that multipacting may be a problem up to about $100 \mathrm{~kW}$ on each window assembly. While this multipacting may assist in cleaning the coaxial metal surfaces, we saw typical vacuum outgassing while multipacting was present. Because of the $100 \mathrm{~kW} /$ window-assembly multipacting, we are planning a change in our configuration that requires fewer than 12 windows. If we stay with 12 windows, we could have multipacting up to the design operating level of 1.2 MW.

Another unexpected occurrence was localized melting at the edges of a few of the coupling irises. Each coupling iris is approximately $1.5 \mathrm{~mm}$ wide by $8.9 \mathrm{~cm}$ long. During the final tuning process, custom-sized holes (about $2 \mathrm{~mm}$ in diameter) were cut at the ends of each slot. After several hours of conditioning at full power levels, we discovered that the edges of some of these holes had melted, but only in the irises with the smaller diameter holes. Simulations with FEA codes indicated a wallcurrent enhancement factor of about 9 or 10 on these smaller holes. This was sufficient to raise a small amount of copper to above the melting temperature.

In most respects however, the conditioning went very well. We saw a few cavity shorts and vacuum bursts in the RFQ cavity. A "blanking box" detects high reflected power, and removes RF drive within a few $\mu$ s, to ensure there is no surface damage from the arc. Conditioning maintained even after the RFQ was 'let up' to atmosphere with dry nitrogen, with subsequent re-conditioning requiring less than twenty minutes. Balancing the conditioning process for simultaneous conditioning of eight windows and the RFQ cavity required much care and learning. Progressive conditioning is a dynamic balance between peak power, duty factor, pulse length, a dc floor level of power, and proper frequency tracking. For successful conditioning, it is imperative to continuously monitor and maintain low pressures in all windows and the RFQ cavity.

\subsection{Beam Commissioning}

Initial beam operation was promising[21], in that we saw good transmission through the entire system on the very first beam pulse on March 16. Output beam energy was right on the design value of $6.7 \mathrm{MeV}$. RFQ transmission and output bunching have the expected behavior as the RF power is varied in the RFQ cavity.

Very limited beam testing during the past week has verified that the several diagnostic devices and systems work as expected. We have measured RFQ transmission, beam energy, acceptance of the injected beam, verified output beam steering and focusing, and confirmed that all sensors and protection systems are functional. Early beams used short pulses $(<1 \mathrm{~ms})$, low repetition rates (5-10 pps) and low currents (<30 mA) (Fig 6) to facilitate debug and tuneup with minimal risk of component damage.

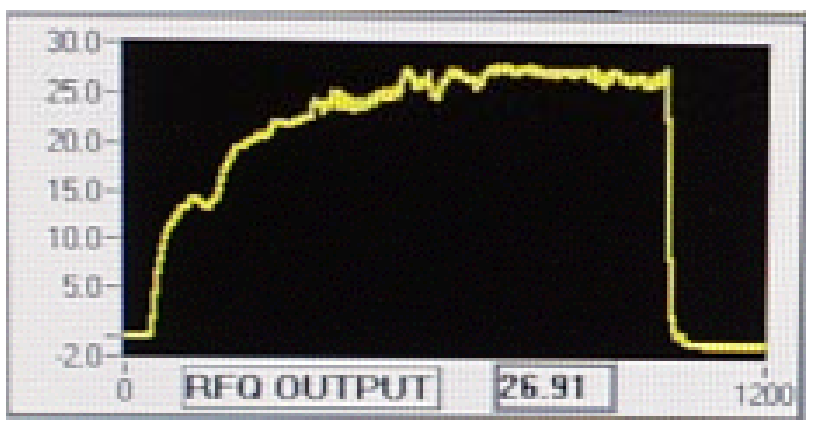

Figure 6. A 1-ms, 27-mA beam pulse from the LEDA RFQ

Low-duty-factor beam-profile measurements are being checked with a two-dimensional wire scanner[22]. Later, video cameras and processing software will be used to measure profiles under $\mathrm{cw}$ conditions. These, and most other diagnostics[23] are mounted in the beam transport line immediately downstream of the RFQ.

Although our critical diagnostics gear[24] is functional, we do not have operating interfaces from all diagnostics into the central control system. Integration and checkout of these systems requires additional beam time. The damaged coupling irises place a limit on the average power we can sustain inside the RFQ, thus we need to run at less-than-ideal cavity power levels. Even though we cannot $\mathrm{cw}$ condition the cavity to the desired $120 \%$ of design power, our pulse conditioning has extended to at least $125 \%$ (1.5-MW) of design level.

During the past week, we have moved from initial beam operation at $5 \mathrm{pps}, 500-\mu \mathrm{s}, 8-\mathrm{mA}$ to reliable operation at $10 \mathrm{pps}, 2.5-\mathrm{ms}$, and $40-\mathrm{mA}$.

We expect that near-future RFQ commissioning will benefit from the successful experience[25] of testing the CRITS RFQ about one year earlier, where beam tests used the LEDA injector and demonstrated up to $100 \mathrm{~mA}$ of $\mathrm{cw}$ proton output beam. For those tests, the injector was operated at $50-\mathrm{keV}$, yet delivered as much as $130 \mathrm{~mA}$ of intput beam. 


\section{SCHEDULE}

Design of the RFQ began in 1995, fabrication in 1996. Assembly was underway in early spring of 1998, and most of the remainder of 1998 was consumed by adding all utilities, support systems and completing final tuning[26]. First high-power conditioning began in late November, and initial beam was introduced in mid-March, 1999.

Beginning in late April, we expect to replace the RF coupling irises with more robust units, then increase our peak and average powers until we are able to sustain cw operation with $100 \mathrm{~mA}$ of proton beam current. At design power levels, the RFQ output beam will have a power of $670 \mathrm{~kW}$.

The coming year will be spent in fully testing the RFQ with beam. Then, we will install a short section of coupled-cavity drift-tube linac (CCDTL) onto the end of the RFQ to permit a complete test of beam matching from the RFQ into the untested CCDTL structure.

Two complete 700-MHz RF systems, including three 1MW klystrons, are in place in the LEDA facility, and are being used for testing components that will be used on the remainder of the LEDA and APT accelerators.

This low-energy beam testing on LEDA will be an important confirmation of the linac design[27] chosen for the APT and similar linacs[28].

\section{LEDA PARTICIPANTS}

Completion of the LEDA hardware involves teaming among several organizations. Some examples include:

- The injector was built using the ion source from Chalk River Labs.

- Initial RFQ beam tests were done with the cw CRITS RFQ, also from Chalk River.

- The vacuum system for the LEDA RFQ was designed, built, and installed by personnel from Lawrence Livermore National Laboratory (LLNL).

- A team from Allied Signal's Kansas City Plant provided the cooling and resonance-control system for the RFQ.

- Development of the beam stop and transport lines from the RFQ to beam stop was completed by teams from General Atomics.

- LANL is teamed with Burns \& Roe, General Atomics, and Westinghouse Savannah River Company for the completion of the APT project.

A project of the size and complexity of LEDA can be successful only through the hard work and excellent collaboration of hundreds of people from many organizations. We extend our sincere thanks to all these teams who helped to make LEDA a success.

\section{REFERENCE}

[1] J. D. Schneider, "APT Accelerator Technology," Proceedings of the 1996 Linac Conf. MO202.

[2] J. Sherman, et al, " A DC Proton Injector for Use in High-Current CW linacs," p. 1424, Proceedings of EPAC98.

[3] T. Zaugg, et al, "Operation of a Microwave Proton Source in Pulsed Mode", LINAC98 Proceedings.

[4] H.V. Smith, et. al, "Comparison of Simulations with Measurements for the LEDA LEBT $\mathrm{H}^{+}$Beam," these conference proceedings.

[5] J. Sherman, et al, "Development and Test Results of the Low-Energy Demonstration Accelerator (LEDA) Proton Injector on a 1.25-MeV cw Radio Frequency Quadrupole," Linac 98 Proceedings.

[6] J. Sherman, et al,'Half-Power Test of a CW Proton Injector Using a 1.25-MeV RFQ," these conference proceedings.

[7] M. Thuot, et al, "A Transient Tolerant Automated Control System for the LEDA 75-keV Injector," these proceedings.

[8] D. Schrage, et al, "CW RFQ Fabrication and Engineering", LINAC98 Proceedings.

[9] R. Floersch \& G. Domer, "Resonance Control Cooling System for the APT/LEDA RFQ," Linac98.

[10] K. Kishiyama, et al, "Testing of Vacuum Pumps for APT/LEDA RFQ," Linac98 Proceedings.

[11] J. Bradley III, et al, "An Overview of the Low-energy Demonstration Accelerator (LEDA) Project RF Systems," PAC97

[12] W. T. Roybal, et al, "LEDA RF Distribution System Design and Component Test Results," LINAC98 Proceedings.

[13] K. Cummings, et al, "Results and Lessons Learned from Conditioning 1-MW CW 350-MHz Coaxial Vacuum Windows," Linac98 Proceedings.

[14] A. H. Regan, "LEDA LLRF Control System Characterization," LINAC98 Proceedings.

[15] A. H. Regan, et al., "APT LLRF Control System Functionality and Architecture," Proc. 1996 Linac Conf. MOP68.

[16] T. H. Van Hagan, D. W. Doll, "Design of an OgiveShaped Beamstop," LINAC98 Proceedings.

[17] W. P. Lysenko \& J. D. Gilpatrick, "High-Energy Beam Transport Beamline for LEDA," Linac98 Proceedings.

[18] J. D. Gilpatrick, et al, "LEDA and APT Beam Diagnostics Instrumentation," PAC97 Proceedings.

[19] M. Thuot, et al, "The Success and Future of EPICS," Proc. 1996 Linac Conf.

[20] L. Young and L. Rybarcyk, "High-Power RF Conditioning of the LEDA RFQ", these conference proceedings.

[21] K. Johnson, "Commissioning of the Low-Energy Demonstration Accelerator (LEDA) Radio-Frequency Quadrupole," these conference proceedings.

[22] J. F. Ohara, et al, "Design and Development of the LEDA Slow-Wire Scanner Profile Measurement," Linac 98 Proceedings.

[23] J. D. Gilpatrick, et al, "LEDA and APT Beam Position Measurements System: Design and Initial Tests," Linac98 Proceedings.

[24] J.D. Gilpatrick, et al., "Low-Energy Demonstration Accelerator (LEDA) Beam Instrumentation: RFQAccelerated Beam Results," these proceedings.

[25] H. V. Smith, et al, "Comparison of Beam Simulations with Measurements for a 1.25-MeV Cw RFQ," Linac98 Proceedings.

[26] L. M. Young \& L. Rybarcyk, "Tuning the LEDA RFQ 6.7-MeV Accelerator," Linac98 Proceedings.

[27] G. P. Lawrence, "High-Power Proton Linac for APT: Status of Design and Development," Linac98 Proceedings.

[28] P. W. Lisowski, "The Accelerator Production of Tritium Project", PAC97 Proceedings. 